\title{
Sur une nouvelle sous-espèce de Niphargus jovanovici Karaman (Crustacea, Amphipoda, Gammaridae) de Dijon, France
}

\author{
Par François Grafi) et Milan Straškraba ${ }^{2}$ )
}

Avec planches 16 (1) et 17 (2)

Les recherches écologiques effectuées sur la faune souterraine aquatique des puits et sources de la ville de Dijon et de sa banlieue, durant les années 1965 et 1966, nous ont permis de récolter et de déterminer 6 espèces déjà connues de Niphargus, à savoir : Niphargus kochianus Bate, N.aquilex Schiödte, N.schellenbergi Karaman ${ }^{3}$ ), N.rhenorhodanensis Schellenberg ${ }^{3}$ ), N.virei Chevreux, N.foreli Humbert et une forme nouvelle voisine de Niphargus jovanovici Karaman.

Nous ne possédons que 4 spécimens de la forme nouvelle, mais étant donné qu'ils présentent plusieurs caractères très nettement aberrants, il convient de donner ci-après la description de cet Amphipode sous le nom de $N$.jovanovici burgundus n.subsp., dénomination se rapportant au lieu de récolte.

Les différentes espèces ont essentiellement été récoltées dans les puits ; la prospection de ce biotope étant facilitée par l'emploi d'un piège (F. Graf 1966). A l'exception de la forme nouvelle et de Niphargus aquilex, les représentants des autres espèces ont été recueillis en grand nombre.

La majorité de ces espèces était déjà connue de Côte-d'Or ; elles sont signalées par P. Paris (1925) sous les noms correspondants à la nomenclature adoptée par E. Chevreux et L. Fage (1925) (N.puteanus de P. Paris étant le N.schellenbergi contemporain, N.plateaui robustus le N.rhenorhodanensis). N.foreli, non mentionné par P. Paris, a été

1) Laboratoire de Biologie Générale, Faculté des Sciences, 2 Boulevard Gabriel. 21 - Dijon. France.

$\left.{ }^{2}\right)$ Laboratoire hydrobiologique de l'Académie des Sciences, Vltavska 17, Smíchov, Praha 5. Tchécoslovaquie.

$\left.{ }^{3}\right)$ Considérée comme espèce indépendante par Straškraba (1966). 
trouvé par R. Husson (A. Schellenberg 1937, R. Husson 1939) dans une mine de fer du département voisin du Doubs ( $N$. foreli thienemanni Schellenberg).

\section{NIPHARGUS JOVANOVICI BURGUNDUS n.subsp.}

Holotype : Mâle de 7,5 mm., récolté à Dijon dans un puits situé dans un jardin particulier au 96, rue de Chenôve.

Collection de François Graf.

Paratypes: Trois mâles de 7,5 mm., 6,5 mm. et $5 \mathrm{~mm}$. en provenance de la même localité, dans des puits situés rue Pierre Boisson.

Collections de François Graf et de Milan Straškraba.

Diagnose : Forme voisine de N.jovanovici jovanovici Karaman, distincte par les caractères suivants :

- dactyles des péréiopodes 5-7 présentant plusieurs épines supplémentaires.

- basipodite du péréiopode 7 élargi.

- uropodes 2 avec épines terminales courtes, correspondant seulement à la moitié de la longueur des branches. La longueur de la branche externe représentant les $3 / 4$ de la branche interne.

- telson garni de 6-7 soies sensorielles aristées sur chaque lobe.

- angle palmaire des gnathopodes avec une forte épine et une épine courte.

Description : Le corps du mâle holotype est grêle. Les plaques coxales sont un peu plus larges que longues, les segments de l'urosome garnis d'une épine de chaque côté, les péréiopodes 5-7 allongés, ceux de la 7 e paire mesurant un peu moins des $3 / 4$ de la longueur du corps.

Les antennes 1 atteignent la moitié de la longueur du corps, leur flagelle principal étant composé de 23 articles ; les bâtonnets hyalins sont aussi longs que les segments correspondants (pl. 1, fig. B). Le flagelle accessoire est constitué de 2 segments, avec une soie sensorielle aristée sur le premier article ; deux soies semblables sont placées à l'extrémité du $3^{\mathrm{e}}$ article basal (pl. 1, fig. A).

Le flagelle des antennes 2 est constitué de 8 articles.

Le $3^{\mathrm{e}}$ article du palpe mandibulaire est allongé, il présente vers le tiers basal un groupe dorsal de 2 longues soies et, en son milieu et au tiers supérieur, deux groupes latéraux de 4 soies qui accompagnent la combe ventrale (pl. 2, fig. M).

La maxillule (pl. 2, fig. N) présente un lobe interne conique terminé par une seule soie et un lobe externe garni de 7 dents, la plus interne portant des dents secondaires (pl. 2, fig. O). Le palpe est court, terminé par 2 soies; il n'atteint pas le sommet du lobe externe. 
Le maxillipède comprend un lobe interne court se terminant près de la base du second article du palpe avec une dent et 4 soies. Le lobe externe atteint la moitié $d u$ second article du palpe (pl. 2, fig. P).

Les propodes des gnathopodes 1 et $\mathbf{2}$ (pl. 2, fig. I et J) ont une palme très inclinée ; le dactyle porte une soie courte. Le bord marginal antérieur du gnathopode 1 présente un groupe de 2 soies alors qu'il n'en existe pas sur le gnathopode 2. Dans l'angle palmaire des deux gnathopodes la forte épine est accompagnée d'une épine courte implantée sur la face interne (pl. 2, fig. K).

Les péréiopodes 3 et 4 ont approximativement la même longueur, correspondant à la moitié de celle du péréiopode 7 (P $3: 2,512 \mathrm{~mm}$. $\mathrm{P} 4: 2,537$ mm. P5:3,150 mm. P6:4,325 mm. P 7:5,037 mm.) (pl. 1, fig. D). L'article basal du péréiopode 7 est élargi, le lobe distal de l'angle postérieur proéminent (pl. 1, fig. D'). Les dactyles 5-7 ont un socle robuste, une griffe courte et 3,5 et 6 épines supplémentaires (pl. 1, fig. G), les dactyles 3-4 portant 2 épines supplémentaires.

Les plaques épimèrales sont rectangulaires, armées de 0-1-1 épines et présentent distalement une forte soie angulaire (pl. 2, fig. L).

Les pléopodes possèdent 2 épines crochues par rétinacle (pl. 1, fig. H).

Les uropodes 1 ont des branches subégales et une forte épine basale (pl. 1, fig. E).

Les branches des uropodes 2 sont inégales, l'externe correspondant aux $3 / 4$ de l'interne (pl. 1, fig. F). Les épines terminales de l'uropode 2 n'atteignent que la moitié de la longueur des branches.

Les uropodes 3 ne sont pas différenciés.

Le telson présente des lobes allongés, chacun d'eux porte distalement 6-7 fortes soies sensorielles aristées et 3 épines courtes (p]. 1, fig. C).

Variabilité : Les spécimens paratypes sont, pour les caractères indiqués dans la description, presque identiques à l'holotype. Le nombre des soies sensorielles aristées de chaque lobe du telson varie de 5 à 7.

Ecologie : Les stations citées précédemment se situent dans le quartier de Larrey, sur la rive gauche de l'Ouche, elles sont distantes d'environ 500 mètres.

Le biotope est une nappe phréatique située vraisemblablement au dessus de celle de la vallée de l'Ouche et localisée dans des graviers würmiens à texture grossière. Les paratypes ont été récoltés au cours du deuxième semestre 1965, l'holotype au début du mois de mars 1966. Les captures ont été faites à l'aide du piège précédemment cité, appâté avec très peu de pain rassis, quelques petits déchets de viande 
de bœuf défraîchie, trois ou quatre feuilles mortes d'Orme, et laissé en place durant quatre à huit jours.

Les récoltes montrent que la nouvelle sous-espèce est peu représentée au milieu d'une abondante population de Niphargus kochianus; avec ces Amphipodes cohabitent le Copépode Eucyclops serrulatus (Fischer) (R. Rouch det.) et l'Isopode Asellus cavaticus puteanus Henry (J-P. Henry 1966).

Affinités : Par Milan Straškraba $\left.{ }^{4}\right)$.

Les caractères principaux de la nouvelle forme, à savoir, les gnathopodes avec la palme très inclinée et une soie dorsale dactylienne, les uropodes 3 non différenciés, le corps grêle, les péréiopodes 7 allongés et les soies sensorielles aristées du telson, montrent l'appartenance à l'espèce $N$.jovanovici sensu lato. Le sous-genre Jovaniphargus de St. Karaman (1960) est identique à cette espèce dans le concept contemporain. Il est évident que le critère de l'espèce n'est basé, pour les Niphargides, que sur des données morphologiques souvent insuffisantes pour établir une distinction entre les groupes d'espèces voisines, les sous-espèces et autres taxons sous-spécifiques. Il est nécessaire d'utiliser les critères zoogéographiques et biologiques, mais cela est souvent limité par la rareté des récoltes. Pour cette raison, nous parlerons de «sous-espèces» avec quelques réserves.

La différence la plus frappante qui sépare notre sous-espèce nouvelle des autres sous-espèces antérieurement décrites est la présence d'un nombre élevé d'épines supplémentaires sur les dactyles des péréiopodes 5-7. Chez plusieurs sous-espèces de $N$.jovanovici les dactyles des péréiopodes sont d'une forme aberrante par rapport aux autres Niphargus, ils sont subchéliformes chez N.j.jovanovici Karaman, N.j.grandii Ruffo (dans ces deux cas, les dactyles 5-7 portent plusieurs groupes de soies dorsales), N.j.bajusaricus (Schellenberg), N.j.kieferi Schellenberg, N.j.multipennatus Sket, et partiellement aussi chez N.j.incertus Dobreanu, Manolache et Puscariu et N.j.ponoricus Dancău. Ce caractère est absent chez N.j.kurdus (Derzhavin) ditécr du Kurdistan comme espèce indépendante et attribué à N.jo-

4) L'auteur tient à remercier le Dr. H.-E. Gruner du «Zoologisches Museum der Humboldt-Universität» d'avoir bien voulu lui confier des $N$.j.jovanovici et $N$.j.kieferi, et le Dr. B. Sket qui lui a adressé des spécimens de $N$.j.multipennatus et l'a aidé très vivement dans la comparaison de cette sous-espèce avec d'autres josanovici. Grâce à l'obligeance du Dr. H. Löffler, l'auteur a pu étudier des exemplaires de N.laisi d'Autriche, et il doit au Dr. J. Vornatscher d'avoir pu observer plusieurs collections de N.j.bajuvaricus de Vienne. 
vanovici par J. A. Birstein (1952) bien qu'il diffère des autres jovanovici par la présence de $2-3$ soies dorsales aux dactyles des gnathopodes. La forme exacte du dactyle est inconnue chez N.j.serbicus Karaman.

Parmi les espèces voisines, N.gallicus Schellenberg et $N$.delamarei Ruffo possèdent de même des dactyles aberrants (chez le dernier, les dactyles sont semblables à ceux de $N$.j.grandii pour le caractère précédemment cité).

Un autre caractère frappant de $N$.j.burgundus est le nombre élevé de soies pennées des lobes du telson, ce caractère rappelle le $N . j . m u l t i$ pennatus de B. Sket.

B. Sket a bien voulu nous adresser un spécimen mâle de $5 \mathrm{~mm}$. de N.j.multipennatus provenant de la localité de Dovjez près de Ljubljana, distante de quelques kilomètres seulement de la localité holotypique. Cette station nouvelle s'ajoute ainsi à celle signalée par M. Meštrov (1960) dans les environs de Zagreb. Grâce à l'obligeance de B. Sket il est possible de comparer les principaux caractères de cette sous-espèce avec burgundus et les autres jovanovici. Les figures 1,2 et 3 adjointes à la planche 1 montrent l'uropode 2 , la forme de la base du péréiopode 7 et le dactyle de cet appendice chez N.j.multipennatus. La forme du $2 \mathrm{e}$ uropode de multipennatus est, comme chez burgundus, aberrante par rapport aux autres sous-espèces de jovanovici. En effet, si chez multipennatus et burgundus la branche externe est de 1/4 plus courte que la branche interne, ces branches sont presque égales chez les autres sous-espèces.

Quoique les caractères des dactyles et du telson soient très nets, et qu'il y ait encore bien d'autres caractères distinctifs utilisés par S. Ruffo (1937) dans la clé des sous-espèces et par D. Dancău (1964) dans une modification récente de cette clé (plusieurs sous-espèces n'y sont malheureusement pas incluses), je suis enclin, après avoir étudié plusieurs sous-espèces de jovanovici, à distinguer deux lignées évolutives de l'espèce basées sur un caractère de haute valeur taxonomique : la forme de la base des péréiopodes 5-7.

Le premier groupe réunit les sous-espèces dont les bases des péréiopodes 5-7 ne présentent pas de lobe postérieur (jovanovici, bajuvaricus, grandii, multipennatus) le second groupant les formes dont le lobe postérieur des basipodites 5-7 est bien développé (serbicus, incertus, kurdus, burgundus). Deux formes ont une position incertaine car leurs basipodites sont étroits mais avec un lobe développé (kieferi, ponoricus). Le N.j.jovanovici de Dobreanu, Manolache et Puscariu (1951) n'est vraisemblablement pas identique au jovanovici de Karaman car il 
présente des bases ovalaires. D'autres caractères, notamment les épines terminales des uropodes 2 plus courtes (correspondant à la moitié de la longueur des branches) et la forme du telson, montrent qu'il s'agit là très probablement de serbicus Karaman (1960).

D. Dancău (1963), se référant aux auteurs précédemment cités, parle d'un groupe jovanovici-laisi-delamarei, et rapproche de ce groupe $N$.gallicus. Ce sont les caractères «palme des gnathopodes inclinée» et "présence d'une soie dorsale au dactyle» qui sont communs à ces espèces ; ces critères n'étant valables que pour le gnathopode 2 de $N$.gallicus et $N$.laisi. Des différences substantielles sont indiquées par les uropodes 3 , différenciés chez $N$.gallicus et formés de 2 articles allongés chez $N$.delamarei, et nous considérons que ce critère permet la différenciation des sous-genres. En effet, la plupart des genres et sous-genres bien définis dans le groupe (Niphargus, Phaenogammarus, Orniphargus, Niphargopsis, Niphargellus) possèdent des uropodes caractéristiques.

En ce qui concerne $N$.laisi, à la suite de l'étude effectuée sur des exemplaires d'Autriche (collection de H. Löffler), les différences observées dans la dentition du lobe externe de la maxille 1 montrent que cette espèce semble être plus proche de N.kochianus que de N.jovanovici. Enfin St. Karaman (1943) considère l'espèce N.parvus comme voisine de laisi, mais cette espèce a des propodes gnathopodiaux différents et les uropodes 3 différenciés.

\section{RÉSUMÉ}

La sous-expèce nouvelle, Niphargus jovanovici burgundus n.subsp., est décrite d'un puits de Dijon, France. Les principaux caractères distinctifs sont données et une comparaison est faite avec les sous-espèces connues. Deux groupes sous-spécifiques sont établis pour $N$.jovanovici et quelques remarques critiques, concernant les rapports de jovanosici avec les autres espèces, sont indiquées.

\section{SUMMARY}

The new subspecies, Niphargus josanovici burgundus n. subsp., is described from a well at Dijon, France. The principal diagnostic characters are given and a comparison with other known subspecies of $N$.jovanovici is made. Two groups of subspecies in $N$. jovanovici are distinguished and some critical remarks on probable affinities of $N$.jovanovici with other species of Niphargus are made. 


\section{B I B LI O GRAP H I E}

Balazuc, J. (1957) - Notes sur les Amphipodes souterrains ; 2 : additions à la faune gallo-rhénane. N. biospéol. 12:75-80.

Birstein, J. A. (1952) - Podzemnye bokoplavy rayona Khosta-Gudauta, Zapadnoe zakavkazie. Bull. Soc. Nat. Moscow 57, (1):26-39 (In Russian).

Chevréux, E., et Fage, L. (1925) - Amphipodes. Faune de France, 9, $488 \mathrm{pp}$. Lechevalier, Paris.

Dancau, D. (1963) - Niphargus gallicus Schell., Amfipod subteran nou pentru fauna R.P.R. Comun. Acad. R.P.R., 13 : 123-129.

- (1964) - Asupra cîtorva amfipode subterane din R. P. Romînă. Niphargus dacicus n.sp. şi Niphargus jovanovici ponoricus n.ssp. Lucr. Inst. Speol. ,Emil Racovitza“", 1-2: 461-474.

Derzhavin, A. N. (1945) - Podzemnye bokoplavy Zakavkazia (The subterranean Amphipoda of Transcaucasus). Bull. Acad. Sci. Azerb. SSR, 8 : 27-43 (In Russian with English summary).

Dobreanu, E., Manolache, C., et Puscariu, V. (1951) - Subspecii de Niphargus jovanovici Karaman in Republica Populara Romana. Comun. Acad. R.P.R., 1 : 579-584.

Graf, F. (1966) - Description d'un piège à Niphargus. International Journal of Speleology, $2: 287-289$.

Henry, J. P. (1967) - Un Aselle phréatique en Bourgogne. Bull. Sc. Bourgogne, 24 (sous presse).

Husson, R. (1939) - Amphipodes des galeries de mines de France. Arch. Zool. expér. géné., $81: 101-111$.

Karaman, Sт. (1931) - 4. Beitrag z. K. der Süßwasseramphipoden. Bull. Soc. Sci. Skoplje, 9, sect. sci. nat., 3: 93-107.

- (1943) - Die unterirdischen Amphipoden Südserbiens. Pos. Izd. 135, prir. i mat. spisi $34: 163-312$.

- (1960) - Weitere Beiträge z. K. der jugoslawischen Niphargiden. Bull. Mus. hist. nat., (B) 15: 75-90.

Meštrov, M. (1960) - Faunistiško-ekološka i biocenološka istraživanja podzemnih voda savske nizine. Biol. Glasnik, 13 : 73-109.

Paris, P. (1925) - La faune cavernicole de la Côte d'Or. C.R. 49e sess. A.F.A.S., Grenoble, 449-450.

Ruffo, S. (1937) - Su alcune specie italiane del gen. Niphargus. Boll. Istit. Entom. Bologna, $9:$ 153-183.

- (1954) - Anfipodi di acque interstiziali raccolti dal Dr. C. Delamare Deboutteville in Francia, Spagna e Algeria. Vie et milieu, 4 : 669-681.

Schellenberg, A. (1932) - Vier blinde Amphipoden in einem Brunnen Oberbayerns. Zool. Anz., 98 : 131-139.

- (1936) - Subterrane Amphipoden Badens, nebst einem neuen Niphargus aus Polen. Zool. Anz., 113: 67-73.

- (1937) - Niphargen (Amphipoda) des Französischen Jura und Jugoslawiens. Zool. Anz., 120:161-169.

Skeт, B. (1957) - Einige neue Formen der Malacostraca (Crust.) aus Jugoslawien. Bull. Scient. Beograd, 3,3: 70-71.

Straškraba, M. (1966) - Amphipoda. Limnofauna Europaea (in litteris). 


\section{EXPLICATIONS DES PLANCHES 16 (1) et 17 (2)}

\section{PLANCHE 16 (1)}

Fig. A à H : Niphargus jovanovici burgundus n.subsp. Holotype.

Fig. A : Flagelle accessoire des antennes.

Fig. B : Article antennaire et bâtonnet hyalin.

Fig. C : Telson.

Fig. D : Péréiopodes 5 (P 5) et 7 (P 7).

Fig. $\mathrm{D}^{\prime}$ : Basipodite 7.

Fig. E : Uropode 1.

Fig. F : Uropode 2.

Fig. G : Dactyles des péréiopodes 5 (d.5), 6 (d.6) et 7 (d. 7).

Fig. $\mathrm{H}$ : Epines crochues du rétinacle.

Fig. 1, 2 et $3:$ N.j.multipennatus Sket.

Fig. 1 : Uropode 2.

Fig. 2 : Basipodite 7.

Fig. 3 : Dactyle 7.

\section{PLANGHE 17 (2)}

Fig. I à P : Niphargus jovanovici burgundus n.subsp. Holotype.

Fig. I : Gnathopode 1.

Fig. J : Gnathopode 2.

Fig. K : Angle palmaire des gnathopodes 1 et 2.

Fig. L : Plaques épimèrales.

Fig. M : Mandibule.

Fig. $\mathrm{N}$ : Maxille 1.

Fig. O : Dentition du lobe externe de la maxille 1.

Fig. $\mathrm{P}$ : Maxillipède. 
SPELEOLOGY III
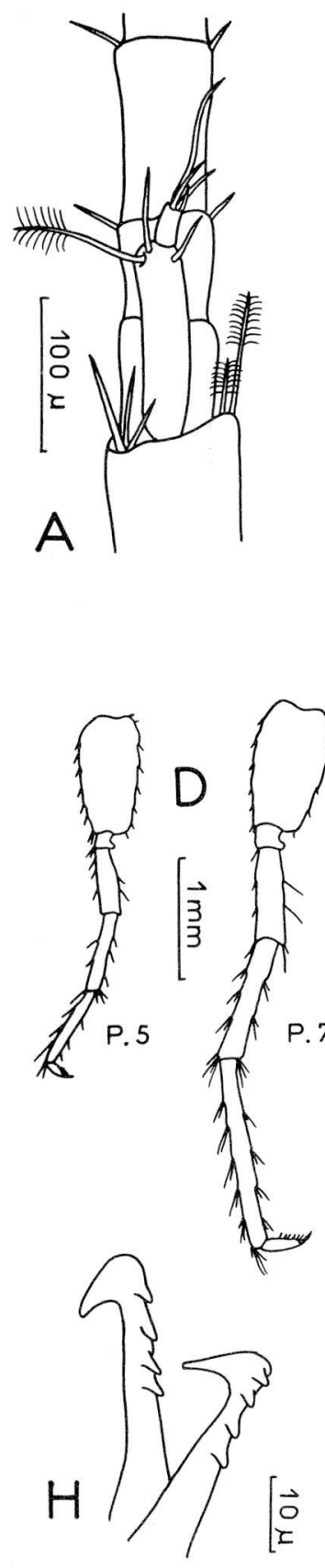

(Graf \& Straskraba, 1)

PLATE 16
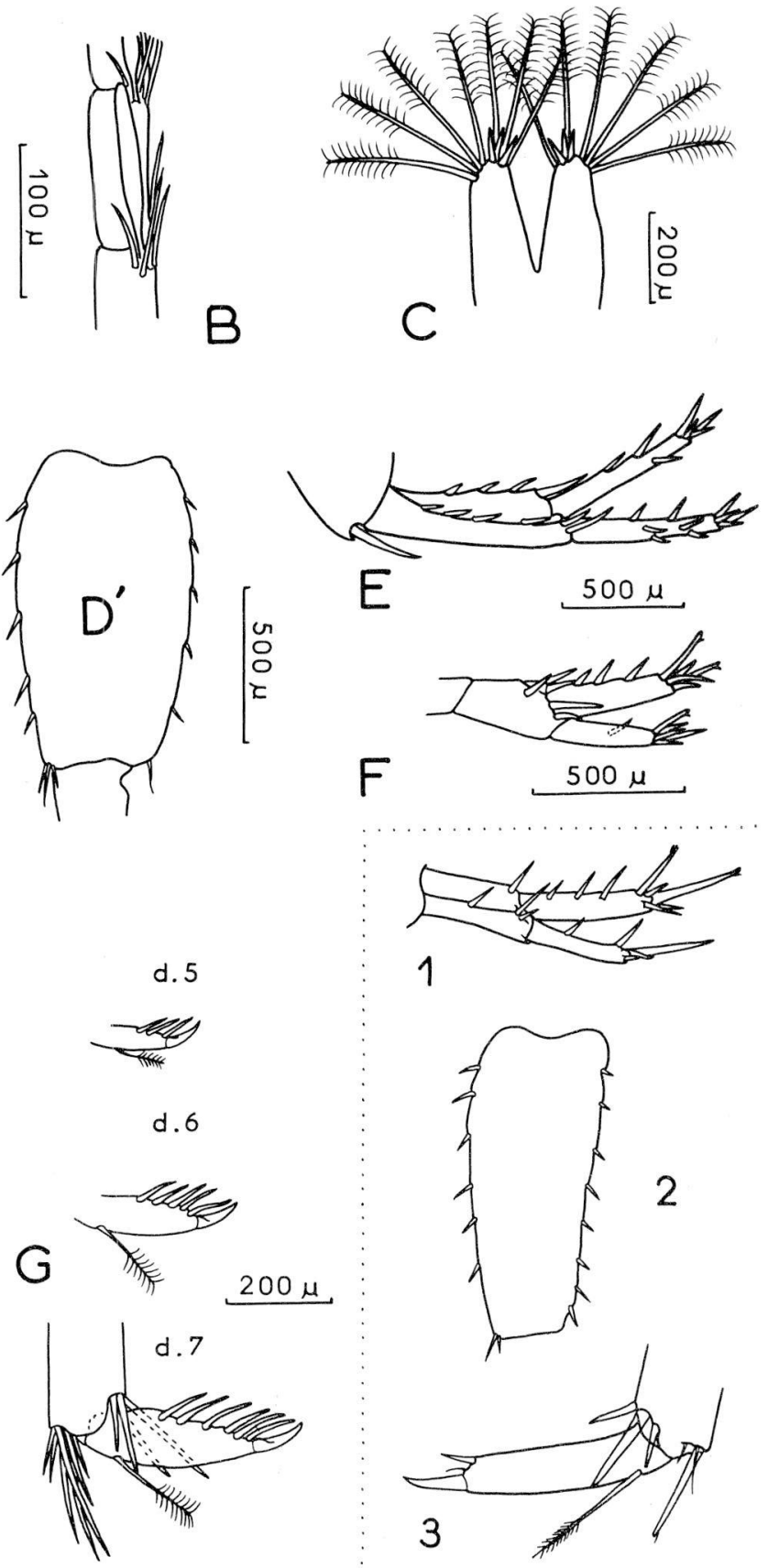

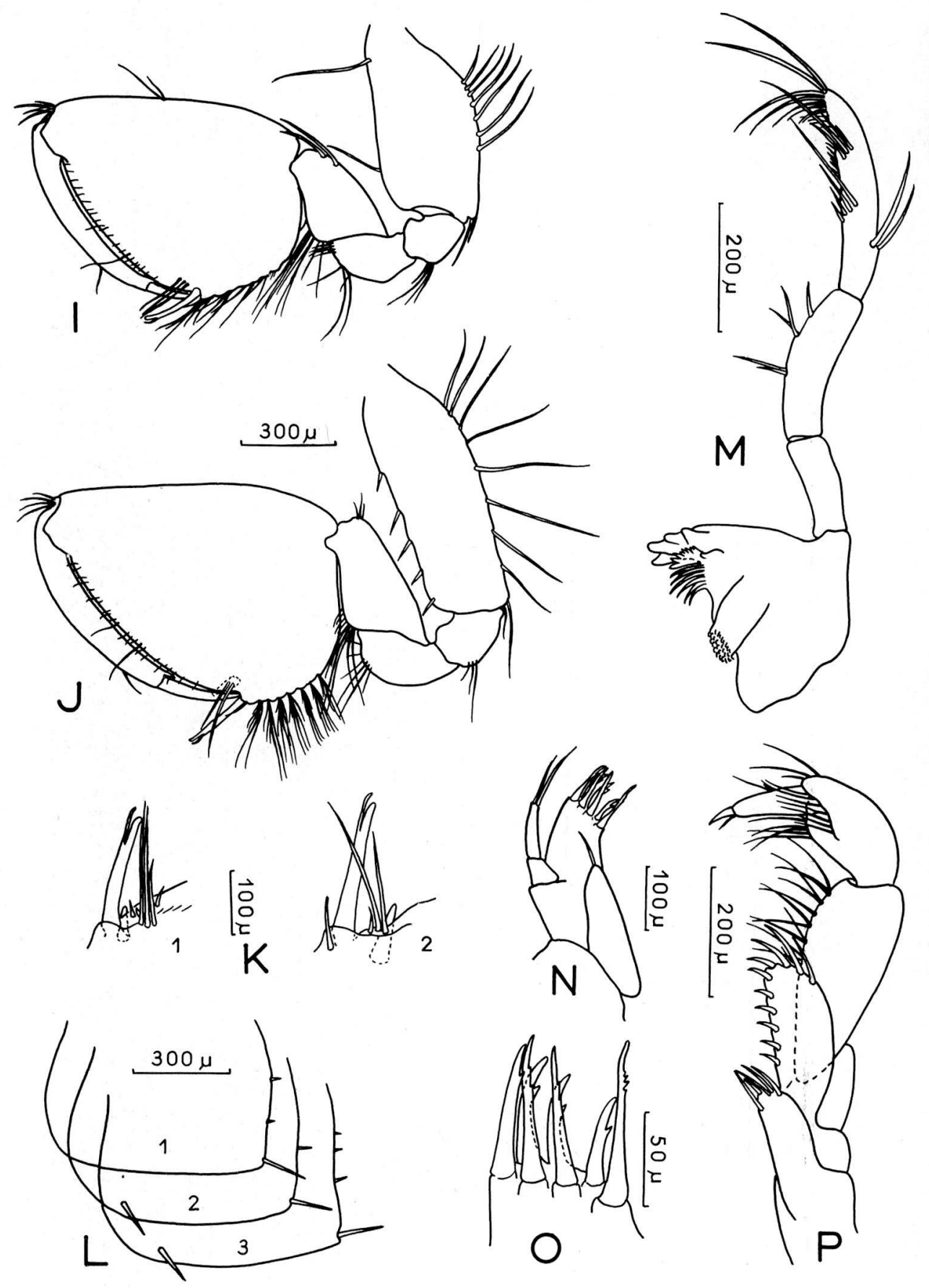